\title{
Measurement and Analysis of the Expected Financial Risks of the Portfolio using Mathematical Methods
}

\author{
Dali Magrakvelidze \\ Georgian Technical University, 0175, Tbilisi, Georgia
}

doi: https://doi.org/10.21467/abstracts.93.16

\begin{abstract}
A risk metric is a measure of an uncertainty in the future value of a portfolio, i.e. a measure of uncertainty in the portfolio's return or profit and loss (P\&L). Its fundamental purpose is to summarize the potential for the deviations from a target or the expected value. Value at risk (VaR) is one of the most important and widely used statistics in economics that measures the potential of the risk of loss. Practically it was accepted as a cornerstone of the risk and common language in basic financial institutions and adjustment management. VaR measures the probability level of the maximum loss for portfolioduring some period of the time. Apart from the traditional way of measuring the loss, VaR method is more adaptive and scientific. During a given period, VaR measures the worst cases of the losses. From Mathematical perspective, VaR corresponds to the precentral of the percent loss of the portfolio and can be expressed as a potential loss of current value of the portfolio, or the loss form the expected value on the horizon.

A number of different methods are available to measure VaR. At the most fundamental level, they separate into local valuation and full valuation. This separation reflects a trade-off between speed of computation and accuracyof valuation. Among local-valuation models, delta-normal models use a combination of the delta or linear exposures with the covariance matrix. Among full-valuation models, historical simulation is the easiest to implement. It uses the recent history of the risk factors to generate hypothetical scenarios, where the full valuation is applied. Finally, the most complete model but also the most difficult to implement is the Monte Carlo simulation approach. This imposes a particular stochastic process for the risk factors, from those the various sample paths are simulated. Full valuation for each sample path generates a distribution of portfolio values.

The Monte Carlo (MC) simulation approach is a parametric method that generates random movements in risk factors from estimated parametric distributions. Positions can be priced using full valuation.MC simulation can incorporate the passage of time, which will create structural changes in the portfolio. This includes the time decay of options; the daily settlement of fixed, floating, or contractually specified cash flows; and the effect of prespecified trading or hedging strategies. These effects are especially important as the time horizon lengthens, which is the case for the measurement of credit risk. The Monte Carlo (MC) simulation approach is a parametric method that generates random movements in risk factors from estimated parametric distributions. Positions can be priced using full valuation. In brief, the method proceeds in two steps.

First, the risk manager specifies a parametric stochastic process for all risk factors. Parameters such as risk and correlations can be derived from historical oroptions data.

Second, fictitious price paths are simulated for all the riskfactors. At each horizon considered, the portfolio is marked to market using full valuation as in the historical simulation. Each of these "pseudo" realizations then is used to compile a distribution of returns, from which a VaR figure can be measured. The method is summarized in Figure 1.
\end{abstract}

(C) 2020 Copyright held by the author(s). Published by AIJR Publisher in "Abstracts of The Second Eurasian RISK-2020 Conference and Symposium" April 12- 19, 2020, Tbilisi, Georgia. Jointly organized by AMIR Technical Services LLC, Georgian Technical University, Institute of Geography (Kazakhstan) and Russian Institute of Petroleum Geology and Geophysics. 
Monte-Carlo method

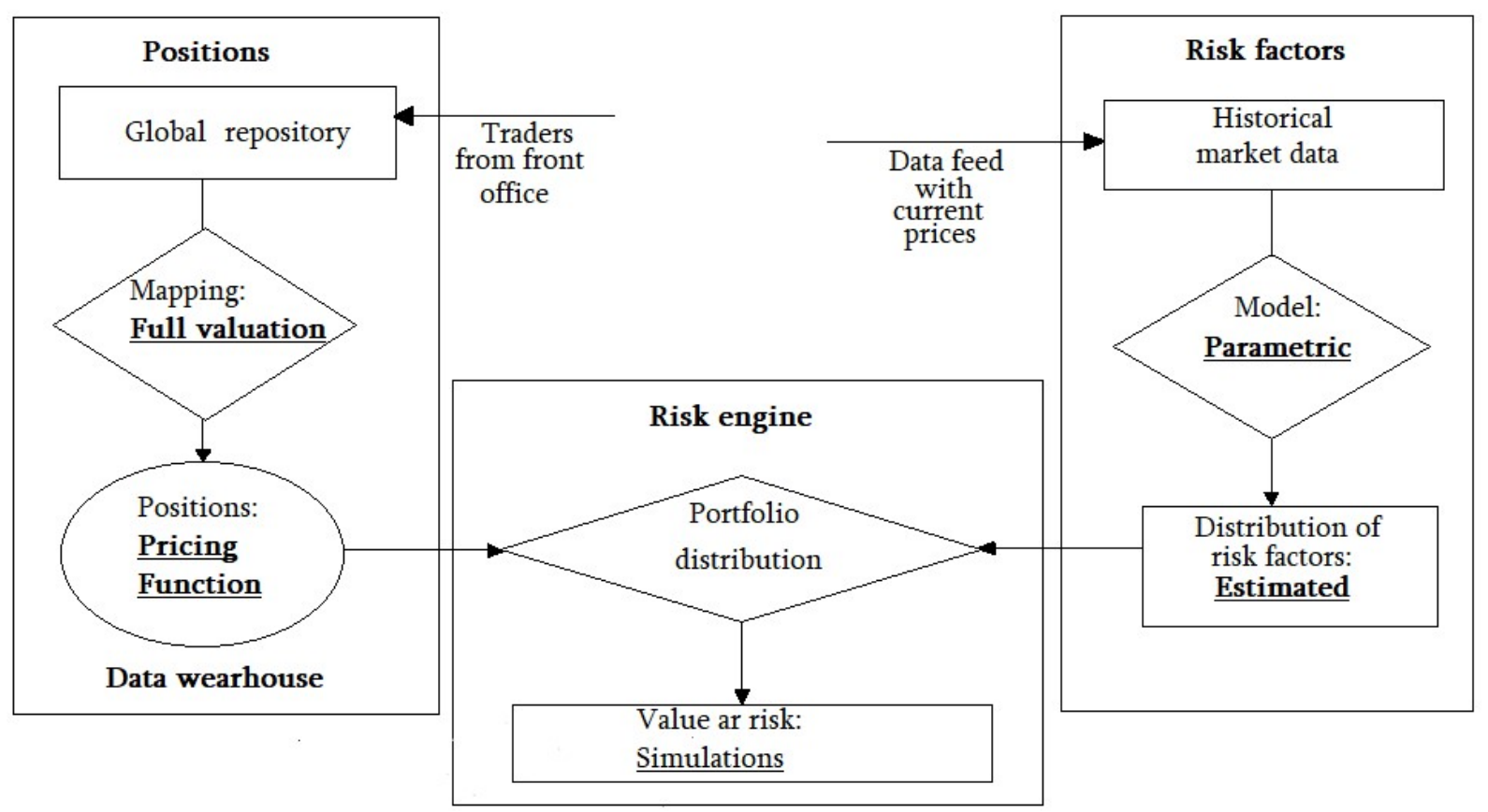

Figure 1. The method's summarize.

Overall, this method probably is the most comprehensive approachin measuring market risk if the modeling is done correctly. This is theonly method that can fully handle financial risks.It can incorporate nonlinear positions, nonnormal distributions, andeven user-defined scenarios 